TRANSACTIONS OF THE

AMERICAN MATHEMATICAL SOCIETY

Volume 355, Number 11, Pages 4581-4594

S 0002-9947(03)03263-X

Article electronically published on July 8, 2003

\title{
MAXIMAL COMPLEXIFICATIONS OF CERTAIN HOMOGENEOUS RIEMANNIAN MANIFOLDS
}

\author{
S. HALVERSCHEID AND A. IANNUZZI
}

\begin{abstract}
Let $M=G / K$ be a homogeneous Riemannian manifold with $\operatorname{dim}_{\mathbb{C}} G^{\mathbb{C}}=\operatorname{dim}_{\mathbb{R}} G$, where $G^{\mathbb{C}}$ denotes the universal complexification of $G$. Under certain extensibility assumptions on the geodesic flow of $M$, we give a characterization of the maximal domain of definition in $T M$ for the adapted complex structure and show that it is unique. For instance, this can be done for generalized Heisenberg groups and naturally reductive homogeneous Riemannian spaces. As an application it is shown that the case of generalized Heisenberg groups yields examples of maximal domains of definition for the adapted complex structure which are neither holomorphically separable nor holomorphically convex.
\end{abstract}

\section{INTRODUCTION}

It is well known that complexifications of a real-analytic manifold $M$ exist and are equivalent near $M$, but differ, usually very much, in nature. If a complete real-analytic metric on $M$ is given, one can construct canonical complexifications of $M$ compatible with the metric by defining an adapted complex structure on a domain $\Omega$ of the tangent bundle $T M$ (see [GS] and [LS]). This structure can be characterized by the condition that the "complexification" $(x+i y) \mapsto y \gamma^{\prime}(x) \in \Omega$ of any geodesic $x \mapsto \gamma(x)$ of $M$ be a complex submanifold near the zero section. By the results of Guillemin-Stenzel and Lempert-Szöke cited above, the adapted complex structure exists and is unique on a sufficiently small neighborhood of $M$. Here $M$ is identified with its zero section in $T M$.

In particular, it is natural to ask for maximal domains around $M$ with an adapted complex structure. By functoriality of the definition these may be regarded as invariants of the metric, i.e., isometric manifolds have biholomorphic maximal domains. For instance, examples are known for symmetric spaces of non-compact type $([\overrightarrow{\mathrm{BHH}}])$, compact normal homogeneous Riemannian spaces $([\mathrm{Sz2}])$, compact symmetric spaces ([Sz1] ) and spaces obtained by Kählerian reduction of these ([A] $)$. Note that in the mentioned cases maximal domains turn out to be Stein.

The aim of this work is to characterize maximal domains for the adapted complex structure for a class of homogeneous Riemannian spaces with "big" isometry group.

Received by the editors December 31, 2001 and, in revised form, November 18, 2002.

2000 Mathematics Subject Classification. Primary 32C09, 53C30, 32M05; Secondary 32 Q28.

Key words and phrases. Adapted complex structure, homogeneous Riemannian spaces, Stein manifold.

The first author was partially supported by SFB 237, "Unordnung und große Fluktuationen".

The second author was partially supported by University of Bologna, funds for selected research topics. 
Let $M=G / K$, with $G$ a Lie group of isometries and $K$ compact, and assume that $\operatorname{dim}_{\mathbb{C}} G^{\mathbb{C}}=\operatorname{dim}_{\mathbb{R}} G$, where $G^{\mathbb{C}}$ is the universal complexification of $G$. Then $K^{\mathbb{C}}$ acts on $G^{\mathbb{C}}$ and the left action on $M$ induces a natural $G$-action on $T M$. Our main result is that, under certain extensibility assumptions on the geodesic flow of $M$, one obtains a real-analytic and $G$-equivariant map $P: T M \rightarrow G^{\mathbb{C}} / K^{\mathbb{C}}$ such that (see Theorem 3.2 for the precise statement)

The connected component of the non-singular locus of DP containing $M$ is the unique maximal domain on which the adapted complex structure exists.

This applies to the case of naturally reductive homogeneous Riemannian spaces (Corollary 3.3) and of generalized Heisenberg groups (see Sections 4 and 5).

As an application, it is shown that for all generalized Heisenberg groups such a maximal domain is neither holomorphically separable, nor holomorphically convex (Proposition 5.1). We are not aware of previous non-Stein examples. In the case of the 3-dimensional Heisenberg group, we determine its envelope of holomorphy as well as a certain maximal Stein subdomain (Proposition 4.3).

\section{Preliminaries}

Here we introduce some notation and briefly recall basic results that we will need in the present paper. Let $M$ be a complete real-analytic Riemannian manifold, which will often be identified with the zero section in its tangent bundle $T M$. Following [LS] (see also [GS] for an equivalent characterization), we say that a realanalytic complex structure defined on a domain $\Omega$ of $T M$ is adapted if all complex leaves of the Riemannian foliation are submanifolds with their natural complex structure, i.e., for any geodesic $\gamma: \mathbb{R} \rightarrow \mathbb{C}$, the induced map $f: \mathbb{C} \rightarrow T M$ defined by $(x+i y) \mapsto y \gamma^{\prime}(x)$ is holomorphic on $f^{-1}(\Omega)$ with respect to the adapted complex structure. Here $y \gamma^{\prime}(t) \in T_{\gamma(t)} M$ is the scalar multiplication in the vector space $T_{\gamma(t)} M$.

The adapted complex structure exists and is unique on a sufficiently small neighborhood of $M$. If $\Omega$ is a domain around $M$ in $T M$ on which it is defined, then we refer to it as an adapted complexification. Sometimes these are called Grauert tubes. For later use we need the following fact.

Lemma 2.1. Let $F: T M \rightarrow \mathbb{C}$ be a real-analytic map that is holomorphic on any complex leaf of TM in a neighbourhood of $M$. Then $F$ is holomorphic on every adapted complexification.

Proof. Following the proof of [Sz1. Prop. 3.2, p. 416], one checks that the restriction of $F$ to $M$ extends to a holomorphic map $\hat{F}$ in a neighbourhood $U$ of $M \subset T M$ where the adapted complex structure $J_{0}$ exists, and, in order to have connected leaves, $U$ may be chosen to be starshaped. We can also assume that for any geodesic $\gamma: \mathbb{R} \rightarrow M$ the map $x+i y \mapsto F\left(y \gamma^{\prime}(x)\right)$ is holomorphic for all $x+i y$ such that $y \gamma^{\prime}(x) \in U$. Now $F=\hat{F}$ on $\gamma(\mathbb{R}) \subset M \subset T M$; therefore, $F=\hat{F}$ on every complex leaf, i.e., on $U$. In particular, $D F \circ J_{0}=i D F$ on $U$, and since all these maps are real-analytic, the statement follows from the identity principle.

A real Lie group $G$ acts on a complex manifold $X$, i.e., $X$ is a $G$-manifold, if there exists a real-analytic surjective map $G \times X \rightarrow X$ given by $(g, x) \mapsto g \cdot x$ such that for fixed $g \in G$ the map $x \mapsto g \cdot x$ is holomorphic and $(g h) \cdot x=g \cdot(h \cdot x)$ for all $h, g \in G$ and $x \in X$. Furthermore, if $\operatorname{dim}_{\mathbb{R}} G=\operatorname{dim}_{\mathbb{C}} G^{\mathbb{C}}$, where $\iota: G \rightarrow G^{\mathbb{C}}$ is the universal complexification of $G$ (see, e.g., $[\mathrm{Ho}]$ ), then $\operatorname{Lie}\left(G^{\mathbb{C}}\right)=\mathfrak{g}^{\mathbb{C}}$, and one 
obtains an induced local holomorphic $G^{\mathbb{C}}$-action by integrating the holomorphic vector fields given by the $G$-action. Here $\mathfrak{g}$ denotes the Lie algebra of $G$.

Let $M=G / K$ be a homogeneous Riemannian manifold with $G$ a connected Lie group of isometries and $K$ compact, and consider the induced $G$-action on $T M$ defined by $g \cdot w:=g_{*} w$ for all $g \in G$ and $w \in T M$. Then if $\Omega$ is a $G$ invariant adapted complexification, as an easy consequence of the definitions $g_{*}$ is a biholomorphic extension of the isometry $g$, i.e., $G \subset \operatorname{Aut}(\Omega)$.

If one assumes that $\operatorname{dim}_{\mathbb{R}} G=\operatorname{dim}_{\mathbb{C}} G^{\mathbb{C}}$, then the natural map $\iota: G \rightarrow G^{\mathbb{C}}$ is an immersion, and from the universality property of the universal complexification $K^{\mathbb{C}}$ of $K$ it follows that the restriction $\left.\iota\right|_{K}$ of $\iota$ to $K$ extends to an immersion $\iota^{\mathbb{C}}: K^{\mathbb{C}} \rightarrow G^{\mathbb{C}}$. Moreover, the subgroup $\iota^{\mathbb{C}}\left(K^{\mathbb{C}}\right)$ acts by right multiplication on $G^{\mathbb{C}}$, and one has a commutative diagram

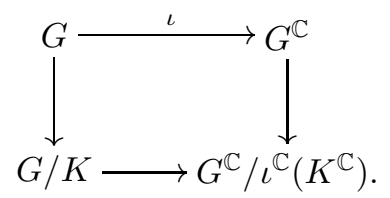

Also notice the $G$-action on $G^{\mathbb{C}} / \iota^{\mathbb{C}}\left(K^{\mathbb{C}}\right)$ defined by $g \cdot h \iota^{\mathbb{C}}\left(K^{\mathbb{C}}\right):=\iota(g) h \iota^{\mathbb{C}}\left(K^{\mathbb{C}}\right)$ for all $g \in G$ and $h \in G^{\mathbb{C}}$.

Lemma 2.2. Let $G$ be a connected Lie group, $K$ a compact subgroup, and assume that $\operatorname{dim}_{\mathbb{C}} G^{\mathbb{C}}=\operatorname{dim}_{\mathbb{R}} G$. Then $G^{\mathbb{C}} / \iota^{\mathbb{C}}\left(K^{\mathbb{C}}\right)$ is a complex $G$-manifold, and $\operatorname{dim}_{\mathbb{C}} G^{\mathbb{C}} / \iota^{\mathbb{C}}\left(K^{\mathbb{C}}\right)=\operatorname{dim}_{\mathbb{R}} G / K$.

Proof. One needs to show that $\iota^{\mathbb{C}}\left(K^{\mathbb{C}}\right)$ is closed in $G^{\mathbb{C}}$. Note that $G^{\mathbb{C}} / \iota^{\mathbb{C}}\left(K^{\mathbb{C}}\right)$ is the orbit space with respect to the $K^{\mathbb{C}}$-action on $G^{\mathbb{C}}$ defined by $k \cdot h:=h \iota\left(k^{-1}\right)$ for all $k \in K^{\mathbb{C}}$ and $h \in G^{\mathbb{C}}$. Since $G^{\mathbb{C}}$ is Stein ([He]) and $K^{\mathbb{C}}$ is reductive, it follows that every fiber of the categorical quotient $G^{\mathbb{C}} \rightarrow G^{\mathbb{C}} / / K^{\mathbb{C}}$ is equivariantly biholomorphic to an affine algebraic variety on which $K^{\mathbb{C}}$ acts algebraically $([\mathrm{Sn}])$. In particular, there exists at least one closed $K^{\mathbb{C}}$-orbit, and consequently $\iota^{\mathbb{C}}\left(K^{\mathbb{C}}\right)$ is closed in $G^{\mathbb{C}}$. Thus $G^{\mathbb{C}} / \iota^{\mathbb{C}}\left(K^{\mathbb{C}}\right)$ is a complex $G$-manifold, and by construction its complex dimension is $\operatorname{dim}_{\mathbb{R}} G / K$.

\section{A characterization of maximal adAPted COMPLEXifications}

If $M=G / K$ is a symmetric space of the non-compact type, then $G^{\mathbb{C}} / K^{\mathbb{C}}$ is a natural candidate for a complexification of $M$, and there exists a $G$-equivariant map $P: T M \rightarrow G^{\mathbb{C}} / K^{\mathbb{C}}$ embedding holomorphically a maximal adapted complexification $\Omega_{M}$ of $M$ (see $\left.[\mathrm{BHH}],[\mathrm{Ha}], \mathrm{AG}\right]$ ). As a matter of fact, one may show that $D P$ is singular on the boundary $\partial \Omega_{M}$ of $\Omega_{M}$.

Here we consider a homogeneous Riemannian manifold $M=G / K$ endowed with the additional data of a certain real-analytic $G$-equivariant map $P$ from $T M$ to a suitable complex $G$-manifold. Proposition 3.1 characterizes a maximal adapted complexification $\Omega_{M}$ as the connected component of $\{D P$ not singular $\}$ containing $M$. Unicity of $\Omega_{M}$ follows.

The existence of such data is proved when $\operatorname{dim}_{\mathbb{C}} G^{\mathbb{C}}=\operatorname{dim}_{\mathbb{R}} G$ and the geodesic flow "extends" holomorphically on $G^{\mathbb{C}} / \iota^{\mathbb{C}}\left(K^{\mathbb{C}}\right)$ (cf. Lemma2.2). As a consequence, the characterization applies to the cases of naturally reductive homogeneous Riemannian spaces and of generalized Heisenberg groups. 
Proposition 3.1. Let $M=G / K$ be an n-dimensional homogeneous Riemannian space and $X$ a G-complex manifold of complex dimension $n$ such that the induced local $G^{\mathbb{C}}$-action is locally transitive. Assume there exists a real-analytic map $P$ : $T M \rightarrow X$ that is

i) G-equivariant and

ii) holomorphic on every complex leaf of $T M$.

Then the connected component $\Omega_{M}$ of $\left\{p \in T M: D P_{p}\right.$ is not singular $\}$ containing $M$ is the unique maximal adapted complexification and $\left.P\right|_{\Omega_{M}}$ is locally biholomorphic.

Proof. First we show that $\Omega_{M}$ is well defined, i.e., $D P$ has maximal rank along $M$. Since from Lemma 2.1 it follows that $P$ is holomorphic on $M$ with respect to the adapted complex structure, this is a consequence of the following fact.

Claim: Assume that $P$ is holomorphic in $p \in T M$. Then $D P_{p}$ has maximal rank.

Proof of the claim. Since $G^{\mathbb{C}}$ acts locally transitively on $X$, there exist elements $\xi_{1}, \cdots, \xi_{n}$ of $\mathfrak{g}$ such that the induced vector fields $\xi_{X, 1}, \cdots, \xi_{X, n}$ on $X$ span a totally real and maximal dimensional subspace $V_{P(p)}$ of $T_{P(p)} X$, where

$$
\xi_{X, j}(x):=\left.\frac{d}{d t}\right|_{0} \exp _{G^{\mathbb{C}}}\left(t \xi_{j}\right) \cdot x
$$

for $j=1, \cdots, n$ and all $x \in X$. By equivariance it follows that $D P_{p}\left(V_{p}\right)=$ $V_{P(p)}$, where $V_{p}$ is the subspace of $T_{p} T M$ spanned by $\xi_{T M, 1}, \cdots, \xi_{T M, n}$; here $\xi_{T M, j}(q):=\left.\frac{d}{d t}\right|_{0} \exp _{G}(t \xi) \cdot q$ for all $q \in T M$. In particular, $\operatorname{dim}_{\mathbb{R}} V_{p}=n$, and since $P$ is holomorphic in $p, V_{p}$ is totally real and $D P_{p}$ has maximal rank, proving the claim.

Now we see that the pulled-back complex structure $J_{o}$ on $\Omega_{M}$ of the complex structure $J$ on $X$ is the adapted complex structure. For this, consider a complex leaf $f: \mathbb{C} \rightarrow T M$ defined by $f(x+i y):=y \gamma^{\prime}(x)$, where $\gamma$ is a geodesic of $M$, and note that by ii)

$$
D P \circ D f(i \eta)=D P \circ J_{o} \circ D f(\eta)
$$

for all $\eta$ tangent in $f^{-1}\left(\Omega_{M}\right)$. Since $D P$ has maximal rank on $\Omega_{M}$, one has

$$
D f(i \eta)=J_{o} \circ D f(\eta)
$$

showing that $J_{o}$ is the adapted complex structure. In particular, $\left.P\right|_{\Omega_{M}}$ is locally biholomorphic.

In order to prove maximality, assume that $J_{o}$ extends analytically in a neighborhood of a certain $p \in \partial \Omega_{M} \subset T M$. By construction, $D P \circ J_{o}=J \circ D P$ on $\Omega_{M}$, and since all maps are real-analytic, $P$ is holomorphic in $p$. Then the above claim shows that $D P_{p}$ has maximal rank, contradicting the definition of $\Omega_{M}$.

Finally we want to show that any adapted complexification $\Omega$ is contained in $\Omega_{M}$. If this is not the case, there exists a point $p$ in $\Omega \cap \partial \Omega_{M}$, and from Lemma 2.1 it follows that $\left.P\right|_{\Omega}$ is holomorphic. In particular, $P$ is holomorphic in $p$, and one obtains a contradiction arguing as above. Thus $\Omega_{M}$ is unique, and this concludes the proof of the statement. 
Now we determine a class of homogeneous Riemannian spaces to which Proposition 3.1 may be applied in order to determine the maximal adapted complexification.

Theorem 3.2. Let $M=G / K$ be a homogeneous Riemannian space with $\operatorname{dim}_{\mathbb{R}} G$ $=\operatorname{dim}_{\mathbb{C}} G^{\mathbb{C}}$, and assume there exists a map $\varphi: \mathbb{C} \times T_{K} M \mapsto \mathfrak{g}^{\mathbb{C}}$, real-analytic and holomophic on the first component, such that $\varphi\left(\mathbb{R} \times T_{K} M\right) \subset \mathfrak{g}$ and $t \mapsto$ $\exp _{G} \circ \varphi(t, v) K$ is the unique geodesic tangent to $v$ at 0 for all $v \in T_{K} M$. Then the map

$$
P: T M \rightarrow G^{\mathbb{C}} / \iota^{\mathbb{C}}\left(K^{\mathbb{C}}\right)
$$

defined by

$$
P\left(g_{*}(v)\right):=\iota(g) \exp _{G^{\mathbb{C}}}(\varphi(i, v)) \iota^{\mathbb{C}}\left(K^{\mathbb{C}}\right)
$$

for all $g \in G$ and $v \in T_{K} M$ is as in Proposition 3.1. In particular, the connected component $\Omega_{M}$ of $\left\{p \in T M: D P_{p}\right.$ is not singular $\}$ containing $M$ is the maximal adapted complexification, and $\left.P\right|_{\Omega_{M}}$ is locally biholomorphic.

Proof. In order to prove that $P$ is well defined, we need to show that if $w=k_{*} v$ for some $k \in K$ and $v \in T_{K} M$, then $P(w)=P\left(k_{*} v\right)$, i.e.,

$$
\exp _{G^{\mathbb{C}}}(\varphi(i, w)) \iota^{\mathbb{C}}\left(K^{\mathbb{C}}\right)=\iota(k) \exp _{G^{\mathbb{C}}}(\varphi(i, v)) \iota^{\mathbb{C}}\left(K^{\mathbb{C}}\right) .
$$

For this, note that $t \mapsto k \exp _{G} \circ \varphi(t, v) K$ is the unique geodesic tangent to $w$ at 0 in $K$; thus,

$$
\exp _{G} \circ \varphi(t, w) K=k \exp _{G} \circ \varphi(t, v) K .
$$

Then the commutativity of the diagram

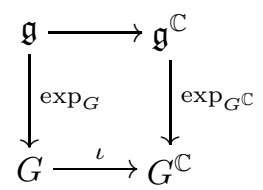

implies that

$$
\exp _{G^{\mathbb{C}}} \circ \varphi(t, w) \iota^{\mathbb{C}}\left(K^{\mathbb{C}}\right)=\iota(k) \exp _{G^{\mathbb{C}}}(\varphi(t, v)) \iota^{\mathbb{C}}\left(K^{\mathbb{C}}\right)
$$

for all $t \in \mathbb{R}$, and equation (1) is a consequence of the identity principle for holomorphic maps.

In order to simplify notation we now assume that the canonical immersion $\iota$ : $G \rightarrow G^{\mathbb{C}}$ is injective, so that once we identify $G$ with $\iota(G)$ the curve $\gamma(t):=$ $\exp _{G^{\mathbb{C}}}(\varphi(t, v)) K$ is the unique geodesic tangent to $v$ at 0 for all $v \in T_{K} M$. In what follows it is easy to check that all arguments apply to the case where $\iota$ is a non-injective immersion.

Fix $x \in \mathbb{R}$, let $g:=\exp _{G^{\mathbb{C}}}(\varphi(x, v))$, and note that

$$
y \mapsto g \exp _{G^{\mathbb{C}}}\left(\varphi\left(y, g_{*}^{-1} \gamma^{\prime}(x)\right)\right) K
$$

is the unique geodesic tangent to $\gamma^{\prime}(x)$ at 0 . Therefore one has

$$
g \exp _{G^{\mathbb{C}}}\left(\varphi\left(y, g_{*}^{-1} \gamma^{\prime}(x)\right)\right) K=\exp _{G^{\mathbb{C}}}(\varphi(x+y, v)) K
$$

for all $y \in \mathbb{R}$, and from the identity principle it follows that

$$
g \exp _{G^{\mathbb{C}}}\left(\varphi\left(z, g_{*}^{-1} \gamma^{\prime}(x)\right)\right) \iota^{\mathbb{C}}\left(K^{\mathbb{C}}\right)=\exp _{G^{\mathbb{C}}}(\varphi(x+z, v)) \iota^{\mathbb{C}}\left(K^{\mathbb{C}}\right)
$$


for all $v \in T_{K} M$ and $z \in \mathbb{C}$. Now for $h \in G, v \in T_{K} M$, let $\gamma, g$ be as above and consider the unique geodesic $\tilde{\gamma}:=h \cdot \gamma$ tangent to $h_{*}(v)$ at 0 . One has

$$
\begin{aligned}
P\left(y \tilde{\gamma}^{\prime}(x)\right) & =P\left(h_{*} y \gamma^{\prime}(x)\right)=h P\left(g_{*} g_{*}^{-1} y \gamma^{\prime}(x)\right) \\
& =h g \exp _{G^{\mathbb{C}}}\left(\varphi\left(i, y g_{*}^{-1} \gamma^{\prime}(x)\right)\right) \iota^{\mathbb{C}}\left(K^{\mathbb{C}}\right) \\
& =h g \exp _{G^{\mathbb{C}}}\left(\varphi\left(i y, g_{*}^{-1} \gamma^{\prime}(x)\right)\right) \iota^{\mathbb{C}}\left(K^{\mathbb{C}}\right) \\
& =h \exp _{G^{\mathbb{C}}}(\varphi(x+i y, v)) \iota^{\mathbb{C}}\left(K^{\mathbb{C}}\right),
\end{aligned}
$$

where we used (2) and the fact that

$$
\exp _{G^{\mathbb{C}}}(\varphi(z, y v)) \iota^{\mathbb{C}}\left(K^{\mathbb{C}}\right)=\exp _{G^{\mathbb{C}}}(\varphi(z y, v)) \iota^{\mathbb{C}}\left(K^{\mathbb{C}}\right)
$$

for all $z \in \mathbb{C}$, since this holds for all $z \in \mathbb{R}$. As a consequence, the map $(x+i y) \mapsto$ $P\left(y \tilde{\gamma}^{\prime}(x)\right)$ is holomorphic for all geodesics $\tilde{\gamma}$ of $M$, i.e., $P$ is holomorphic on every complex leaf of $T M$.

Finally, the map $P$ is $G$-equivariant by construction, and the $G$-action on $G^{\mathbb{C}} / \iota^{\mathbb{C}}\left(K^{\mathbb{C}}\right)$ induces a holomorphic $G^{\mathbb{C}}$-action, which may be obtained through left multiplication on $G^{\mathbb{C}}$. Thus it is obviously transitive, and this yields the statement.

Now let $M$ be a naturally reductive homogeneous Riemannian space, and let $M=G / K$ be a natural realization of $M$, i.e., there exists a reductive decomposition $\mathfrak{g}=\operatorname{Lie}(K) \oplus \mathfrak{m}$ of the Lie algebra of $G$ such that every geodesic in $M$ is the orbit of a one-parameter subgroup of $G$ generated by an element of $\mathfrak{m}$ (see, e.g., [BTV]). Consider the natural projection $\Pi: G \rightarrow M$ and note that $D \Pi_{e}(\mathfrak{m})=T_{K} M$, where $e$ is the neutral element of $G$. Denote by $L: T_{K} M \rightarrow \mathfrak{m}$ the inverse of the restriction of $D \Pi_{e}$ to $\mathfrak{m}$. Since $L$ is linear, it extends $\mathbb{C}$-linearly from $\left(T_{K} M\right)^{\mathbb{C}}$ to $\mathfrak{m}^{\mathbb{C}}$, and the map $\varphi: \mathbb{C} \times T_{K} M \rightarrow \mathfrak{g}^{\mathbb{C}}$ defined by $\varphi(z, v):=z L(v)$ is as in the above theorem. Therefore one has

Corollary 3.3. Let $M=G / K$ be a natural realization of a naturally reductive homogeneous Riemannian space, and assume that $\operatorname{dim}_{\mathbb{R}} G=\operatorname{dim}_{\mathbb{C}} G^{\mathbb{C}}$. Then the map

$$
P: T M \rightarrow G^{\mathbb{C}} / \iota\left(K^{\mathbb{C}}\right)
$$

defined by

$$
P\left(g_{*}(v)\right):=\iota(g) \exp _{G^{\mathbb{C}}}(i L(v)) \iota\left(K^{\mathbb{C}}\right)
$$

for all $g \in G$ and $v \in T_{K} M$ meets the conditions of Proposition 3.1. In particular, the connected component $\Omega_{M}$ of $\left\{p \in T M: D P_{p}\right.$ is not singular $\}$ containing $M$ is the maximal adapted complexification, and $\left.P\right|_{\Omega_{M}}$ is locally biholomorphic.

\section{The 3-Dimensional Heisenberg group}

Here we apply results of the previous section in order to give a concrete description of the unique maximal adapted complexification for the 3-dimensional Heisenberg group. It turns out that such a domain is neither holomorphically separable nor holomorphically convex. We also determine its envelope of holomorphy and a particular maximal Stein subdomain. We remark that in all previous examples of which we are aware maximal adapted complexifications are Stein. 
Consider the 3-dimensional Heisenberg group defined as a subgroup of $G L_{3}(\mathbb{R})$ by

$$
H:=\left\{\left(\begin{array}{ccc}
1 & \alpha & \gamma \\
0 & 1 & \beta \\
0 & 0 & 1
\end{array}\right): \alpha, \beta, \gamma \in \mathbb{R}\right\}
$$

fix the inner product of the tangent space $T_{e} H$ in the neutral element $e$ for which the canonical basis determined by the global natural chart $(\alpha, \beta, \gamma)$ is orthonormal, and let $(a, b, c)$ be coordinates of $T_{e} H$ with respect to this basis. Endow $H$ with the induced $H$-invariant metric

$$
(\mathrm{d} \alpha)^{2}+(\mathrm{d} \beta)^{2}+(\mathrm{d} \gamma-\alpha \cdot \mathrm{d} \beta)^{2}
$$

let $\mathfrak{h}=\operatorname{Lie}(H)$ and define $\varphi: \mathbb{R} \times T_{e} H \rightarrow \mathfrak{h}$ by

$$
\begin{aligned}
\varphi(t,(a, b, c)):=\left(a \frac{\sin (t c)}{c}-b \frac{1-\cos (t c)}{c}, b \frac{\sin (t c)}{c}+a \frac{1-\cos (t c)}{c},\right. \\
\left.\left(t+\frac{a^{2}+b^{2}}{2 c^{2}}\left(t-\frac{\sin (t c)}{c}\right)\right) c\right),
\end{aligned}
$$

where the coordinates of $\mathfrak{h}$ are induced by those of $T_{e} H$ via the natural identification $\mathfrak{h} \cong T_{e} H$. Note that all singularities are removable, and consequently $\varphi$ is real-analytic. Following [BTV the theorem on p.31], one checks that $t \mapsto$ $\exp _{H} \circ \varphi(t,(a, b, c))$ is the unique geodesic tangent to $(a, b, c)$ at 0 . Furthermore, by expanding the power series it is easy to verify that $\varphi(\cdot,(a, b, c))$ extends holomorphically on $\mathbb{C}$ to $\left(T_{e} H\right)^{\mathbb{C}}$, and by considering the polar decomposition $H \times \mathfrak{h} \rightarrow H^{\mathbb{C}}$ of $H^{\mathbb{C}}$ given by $(g, \xi) \mapsto g \exp _{H^{\mathbb{C}}}(i \xi)$, one obtains real-analytic functions $(a, b, c) \mapsto h_{(a, b, c)} \in H$ and $(a, b, c) \rightarrow \xi_{(a, b, c)} \in \mathfrak{h}$ such that

$$
\exp _{H^{\mathbb{C}}} \circ \varphi(i,(a, b, c))=h_{(a, b, c)} \exp _{H^{\mathbb{C}}}\left(i \xi_{(a, b, c)}\right) .
$$

Define $P: T H \rightarrow H^{\mathbb{C}} \cong H \times \mathfrak{h}$ by

$$
g_{*}(a, b, c) \mapsto g \exp _{H^{\mathbb{C}}} \circ \varphi(i,(a, b, c)) \cong\left(g h_{(a, b, c)}, \xi_{(a, b, c)}\right) .
$$

Then Theorem 3.2 implies that the connected component $\Omega_{H}$ containing $H$ of $\{D P$ not singular $\}$ is the maximal adapted complexification. Note that since $P$ is $H$-equivariant, $\Omega_{H}$ is $H$-invariant. Moreover, $T_{e} H$ is a global slice for the $H$ action on $T H$, i.e., the map $H \times T_{e} H \rightarrow T H$ given by $(g,(a, b, c)) \rightarrow g_{*}(a, b, c)$ is an $H$-equivariant real-analytic diffeomorphism; thus, $\Omega_{H}$ is completely determined by its slice $\Omega_{H} \cap T_{e} H$.

Furthermore, since $H$ acts freely on the first component of $H \times \mathfrak{h}$, then the $H$-equivariance of $P$ implies that $D P_{g_{*}(a, b, c)}$ has maximal rank if and only if $D \tilde{P}_{(a, b, c)}$ has maximal rank, where $\tilde{P}:=\left.p_{2} \circ P\right|_{T_{e} H}: T_{e} H \rightarrow \mathfrak{h}$ is given by

$$
\begin{aligned}
\tilde{P}(a, b, c) & =\xi_{(a, b, c)} \\
& =\left(a \frac{\sinh (c)}{c}, b \frac{\sinh (c)}{c},\left(1+\frac{a^{2}+b^{2}}{2 c^{3}}(c-\sinh (c) \cosh (c))\right) c\right) .
\end{aligned}
$$

Here $p_{2}: H \times \mathfrak{h} \rightarrow \mathfrak{h}$ is the canonical projection. It follows that $\Omega_{H}=H \cdot O_{0}$, where $O_{0}$ is the connected component of $\{\operatorname{det}(D \tilde{P}) \neq 0\}$ containing 0 in $T_{e} H$. Now a straightforward computation shows that

$$
\operatorname{det}\left(D \tilde{P}_{(a, b, c)}\right)=\frac{\sinh (c)}{c}\left(\frac{\sinh (c)}{c}+\left(a^{2}+b^{2}\right)\left(\frac{\sinh (c)-c \cosh (c)}{c^{3}}\right)\right)
$$


and therefore

$$
O_{0}=\left\{(a, b, c) \in T_{e} H: a^{2}+b^{2}<\frac{c^{2} \sinh (c)}{c \cosh (c)-\sinh (c)}\right\} .
$$

We want to discuss injectivity of $\left.P\right|_{\Omega_{H}}: \Omega_{H} \rightarrow H^{\mathbb{C}} \cong H \times \mathfrak{h}$, and again this is equivalent to injectivity of $\left.\tilde{P}\right|_{O_{0}}$.

Note that $\tilde{P}$ is equivariant with respect to rotations around the $c$-axis as well as to the reflection $\sigma$ with respect to the plane $\{c=0\}$. In particular, for any

$$
(a, b, c) \in\left\{1+\frac{a^{2}+b^{2}}{2 c^{3}}(c-\sinh (c) \cosh (c))=0\right\},
$$

one has $\tilde{P}(a, b, c)=\tilde{P}(a, b,-c)=\left(a \frac{\sinh (c)}{c}, b \frac{\sinh (c)}{c}, 0\right)$. Therefore we are induced to investigate the domain

$$
O_{1}:=\left\{(a, b, c) \in T_{e} H: a^{2}+b^{2}<\frac{2 c^{3}}{\sinh (c) \cosh (c)-c}\right\} .
$$

Lemma 4.1. The domain $O_{1}$ is the maximal $\sigma$-invariant subdomain of $O_{0}$ containing 0 on which $\tilde{P}$ is injective. In particular, $\left.\tilde{P}\right|_{O_{0}}$ is not injective.

Proof. Let $f_{j}$ be the real function defining

$$
O_{j}=\left\{(a, b, c) \in T_{e} H: a^{2}+b^{2}<f_{j}(c)\right\}
$$

for $j=0,1$. First we want to show that $O_{1}$ is a subdomain of $O_{0}$, i.e.,

$$
f_{1}(c) \leq f_{0}(c)
$$

for all $c \in \mathbb{R}$, which is equivalent to

$$
2 \cosh (c) \leq \frac{\sinh (c)}{c}+\frac{\sinh ^{2}(c)}{c^{2}} \cosh (c)
$$

Expanding in power series, one obtains

$$
\begin{aligned}
& 2+c^{2}+\frac{1}{12} c^{4}+\cdots \\
& \leq\left(1+\frac{1}{6} c^{2}+\frac{1}{120} c^{4}+\cdots\right)+\left(1+\frac{5}{6} c^{2}+\left(\frac{5}{6}+\frac{1}{24}+\cdots\right) c^{4}+\cdots\right) .
\end{aligned}
$$

All coefficients are non-negative, and one easily checks that for $k \geq 2$ the coefficient of $c^{2 k}$ in the last series on the right side is strictly greater than that in the series on the left; hence $O_{1} \subset O_{0}$. Moreover, $\partial O_{1} \cap \partial O_{0}=\left\{(a, b, 0) \in T_{e} H: a^{2}+b^{2}=3\right\}$, and thus $O_{1}$ is a proper subdomain of $O_{0}$.

Furthermore, by the previous remarks, any $\sigma$-invariant domain containing 0 on which $\tilde{P}$ is injective is necessarily contained in $O_{1}$.

Assume that there exist $\left(a^{\prime}, b^{\prime}, c^{\prime}\right),\left(a^{\prime \prime}, b^{\prime \prime}, c^{\prime \prime}\right) \in O_{1}$ such that $\tilde{P}\left(a^{\prime}, b^{\prime}, c^{\prime}\right)=$ $\tilde{P}\left(a^{\prime \prime}, b^{\prime \prime}, c^{\prime \prime}\right)=:(A, B, C)$. If $C=0$, then $c^{\prime}=c^{\prime \prime}=0$, and consequently $a^{\prime}=$ $a^{\prime \prime}=A$ and $b=b^{\prime \prime}=B$. If $C \neq 0$, by eventually acting with $\sigma$ and a rotation around the $c$-axis we may assume that $a, A \geq 0, b=B=0$ and $c>0$. Now one has

$$
a^{\prime} \frac{\sinh \left(c^{\prime}\right)}{c^{\prime}}=a^{\prime \prime} \frac{\sinh \left(c^{\prime \prime}\right)}{c^{\prime \prime}}=A .
$$


Therefore $\left(a^{\prime}, 0, c^{\prime}\right)$ and $\left(a^{\prime \prime}, 0, c^{\prime \prime}\right)$ lie on the same level curve $\rho_{A}: \mathbb{R} \rightarrow T_{e} H$ given by

$$
\rho_{A}(t):=\left(A \frac{t}{\sinh (t)}, 0, t\right) .
$$

One has the following assertion.

Claim: Let $A \geq 0$ and $t_{0} \in \mathbb{R}^{\geq 0}$ be such that $\rho_{A}\left(t_{0}\right) \in \bar{O}_{0}$. Then $\rho_{A}(t) \in O_{0}$ for all $t>t_{0}$.

Proof of the claim. One needs to show that $A^{2} \frac{t^{2}}{\sinh ^{2}(t)}<f_{0}(t)$ for all $t>t_{0}$; that is,

$$
A^{2}<\frac{\sinh ^{2}(t)}{t^{2}} f_{0}(t)
$$

By expanding in power series as above one has the estimate

$$
2 t \cosh ^{2}(t)-3 \cosh (t) \sinh (t)+t>0
$$

for all $t>0$, which by a straightforward computation implies that the derivative of the function on the right-hand side of (31) is positive for all $t>0$, proving the claim.

Now let $t_{0}:=\min \left(c^{\prime}, c^{\prime \prime}\right)$ and note that since $O_{1} \subset O_{0}$, as a consequence of the above claim there exists $\epsilon>0$ such that $\rho_{A}(t) \in O_{0}$ for $t>t_{0}-\epsilon$. In particular, $\left(a^{\prime}, 0, c^{\prime}\right)$ and $\left(a^{\prime \prime}, 0, c^{\prime \prime}\right)$ lie in the same connected real one-dimensional submanifold $N:=\rho_{A}\left(t_{0}-\epsilon, \infty\right)$ of $O_{0}$, and $\left.\tilde{P}\right|_{N}: N \rightarrow\left\{(A, 0, \cdot) \in T_{e} H\right\} \cong \mathbb{R}$ is locally diffeomorphic. Then a classical argument implies that $\left.\tilde{P}\right|_{N}$ is injective; thus $\left(a^{\prime}, 0, c^{\prime}\right)=\left(a^{\prime \prime}, 0, c^{\prime \prime}\right)$, as wished.

We also want to determine the image of $\left.P\right|_{\Omega_{H}}$ in $H^{\mathbb{C}}$. Note that $P\left(\Omega_{H}\right)$ is $H$ invariant, and the polar decomposition implies that $\exp _{H^{\mathbb{C}}}(i \mathfrak{g})$ is a global slice for the $H$-action on $H^{\mathbb{C}}$. Then this can be achieved by describing $\exp _{H^{\mathbb{C}}}\left(i \tilde{P}\left(O_{0}\right)\right)=$ $P\left(\Omega_{H}\right) \cap \exp _{H^{\mathrm{C}}}(i \mathfrak{g})$.

Lemma 4.2. $\tilde{P}\left(O_{0}\right)=\mathfrak{h} \backslash\left\{(A, B, C) \in \mathfrak{h}: A^{2}+B^{2}=3, C=0\right\}$.

Proof. Let $(a, 0, c) \in\left\{a^{2}=f_{1}(c)\right\} \subset \partial O_{1}$ with $c>0$. From the proof of Lemma 4.1 it follows that $(a, 0, c) \in O_{0}$. Since $\tilde{P}(a, 0, c)=\left(a \frac{\sinh (c)}{c}, 0,0\right)$ and

$$
\sqrt{f_{1}(0)}=\sqrt{3} \quad \text { and } \quad \lim _{c \rightarrow \infty} \sqrt{f_{1}(c)} \frac{\sinh (c)}{c}=\infty
$$

it follows that $(A, 0,0) \in \tilde{P}\left(O_{0}\right)$ for all $A>\sqrt{3}$.

For $A>\sqrt{3}$ let $(a, 0, c) \in O_{0}$ be such that $\tilde{P}(a, 0, c)=(A, 0,0)$. By the claim in Lemma 4.1 one has $\rho_{A}(t) \in O_{0}$ for all $t \geq c$. Moreover, one sees that

$$
\tilde{P}\left(\rho_{A}(t)\right)=\left(A, 0, C_{A}(t)\right) \quad \text { with } \quad \lim _{t \rightarrow \infty} C_{A}(t)=\infty .
$$

Then by $\sigma$-invariance of $O_{0}$ and $\sigma$-equivariance of $\tilde{P}$ it follows that $(A, 0, C) \in$ $\tilde{P}\left(O_{0}\right)$ for all $C \in \mathbb{R}$ and $A>\sqrt{3}$.

Now note that $\tilde{P}(a, 0,0)=(a, 0,0)$ and $f_{0}(0)=3$; thus $(A, 0,0) \in \tilde{P}\left(O_{0}\right)$ for all $A<\sqrt{3}$, and, arguing as above, it follows that $(A, 0, C) \in \tilde{P}\left(O_{0}\right)$ for all $C \in \mathbb{R}$ and $A<\sqrt{3}$. 


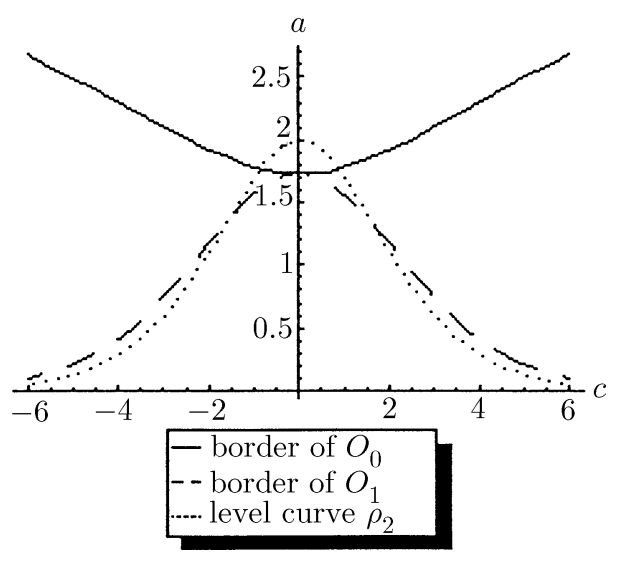

FIGURE 1.

Finally, $\rho_{\sqrt{3}}(0) \in \partial O_{0}$. Thus $\rho_{\sqrt{3}}(t) \in O_{0}$ for all $t>0$. It follows that $t \mapsto \tilde{P}\left(\rho_{\sqrt{3}}(t)\right)$ is injective for $t>0$, and since

$$
\lim _{t \rightarrow 0^{+}} C_{\sqrt{3}}(t)=0 \quad \text { and } \quad \lim _{t \rightarrow \infty} C_{\sqrt{3}}(t)=\infty
$$

one has $(\sqrt{3}, 0, C) \in \tilde{P}\left(O_{0}\right)$ if and only if $C \neq 0$.

The statement follows from the invariance of $O_{0}$ and the equivariance of $\tilde{P}$ with respect to the group of rotations around the $c$-axis.

In Figure 1 one sees the boundary of $O_{0}$ and $O_{1}$ defined by $f_{0}$ and $f_{1}$ respectively as well as the level curve $\rho_{2}$ in the upper half-plane $\{b=0, a \geq 0\}$ of $T_{e} H$. Since $O_{0}$ and $O_{1}$ are invariant with respect to rotations around the $c$-axis, this completely determines their shape and, by $H$-equivariance, that of $\Omega_{H}$.

Proposition 4.3. The maximal domain $\Omega_{H}$ is neither holomorphically separable, nor holomorphically convex. Its envelope of holomorphy is biholomorphic to $\mathbb{C}^{3}$. The maximal $\sigma$-invariant Stein subdomain of $\Omega_{H}$ is biholomorphic to $\left\{\left(z_{1}, z_{2}, z_{3}\right)\right.$ $\left.\in \mathbb{C}^{3}:\left(\operatorname{Im} z_{1}\right)^{2}+\left(\operatorname{Im} z_{2}\right)^{2}<3\right\}$.

Proof. Note that the elements of $H$ with integer entries determine a discrete cocompact subgroup $\Gamma$ of $H$, and from [GH] it follows that $H^{\mathbb{C}} / \Gamma$ is Stein (in fact it is easy to check that $H^{\mathbb{C}} / \Gamma$ is biholomorphic to $\left.\left(\mathbb{C}^{*}\right)^{3}\right)$. Then the proposition on p. 543 of [CIT] applies to show that any holomorphically separable Riemann $H$-domain over $H^{\mathbb{C}}$ is univalent. Moreover, Lemma 4.11implies that $\left.P\right|_{\Omega_{H}}: \Omega_{H} \rightarrow$ $H^{\mathbb{C}}$ is not injective. Therefore $\Omega_{H}$ is not holomorphically separable.

By a result of Loeb ([L, the theorem on p. 186]), a Stein $H$-invariant domain $U$ of $H^{\mathbb{C}}$ is "geodesically" convex, i.e., it is convex with respect to all curves of the form $t \mapsto g \exp _{H^{\mathbb{C}}}(i t \xi)$ with $g \in U$ and $\xi \in \mathfrak{h}$. Since $H^{\mathbb{C}}$ admits polar decomposition and $U$ is $H$-invariant, it is enough to consider curves of the form $\exp _{H^{\mathbb{C}}}(i \eta) \exp _{H^{\mathbb{C}}}(i t \xi)$ with $\exp _{H^{\mathbb{C}}}(i \eta) \in U$ and $\xi \in \mathfrak{h}$. Furthermore, for a two-step 
nilpotent Lie group, one has

$$
\begin{aligned}
\exp _{H^{\mathbb{C}}}(i \eta) \exp _{H^{\mathbb{C}}}(i t \xi) & =\exp _{H^{\mathbb{C}}}\left(i \eta+i t \xi-\frac{t}{2}[\eta, \xi]\right) \\
& =\exp _{H^{\mathbb{C}}}\left(-\frac{t}{2}[\eta, \xi]\right) \exp _{H^{\mathbb{C}}}(i(\eta+t \xi)),
\end{aligned}
$$

and, using $H$-invariance one more time, we conclude that if $U=H \cdot \exp _{H^{\mathbb{C}}}(i D)$, with $D$ a domain in $\mathfrak{h}$, is Stein, then $D$ is convex in the usual affine sense.

Since $P\left(\Omega_{H}\right)=H \cdot \exp _{H^{\mathrm{C}}}\left(i \tilde{P}\left(O_{0}\right)\right)$ and, as a consequence of Lemma 4.2 the domain $\tilde{P}\left(O_{0}\right)$ is not convex, it follows that $P\left(\Omega_{H}\right)$ is not Stein. Now $H^{\mathbb{C}}$ is Stein and $\left.P\right|_{\Omega_{H}}$ is locally biholomorphic. Therefore by $[\underline{\mathrm{R}}$ there exists a commutative diagram

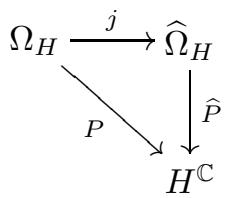

where $\hat{\Omega}_{H}$ is the envelope of holomorphy of $\Omega_{H}$. Moreover, $H$ acts on $\hat{\Omega}_{H}$ and all maps are $H$-equivariant. Furthermore, $\hat{P}$ is injective by the proposition on p. 543 of [CIT, and if $\Omega_{H}$ is holomorphically convex, then $j$ is surjective, and consequently $\hat{\Omega}_{H}$ is biholomorphic to $P\left(\Omega_{H}\right)$, giving a contradiction. Hence $\Omega_{H}$ is not holomorphically convex.

Notice that $\hat{\Omega}_{H} \cong \hat{P}\left(\hat{\Omega}_{H}\right)$ contains $P\left(\Omega_{H}\right)=H \cdot \exp _{H^{\mathbb{C}}}\left(i \tilde{P}\left(O_{0}\right)\right)$ and the convex envelope of $\tilde{P}\left(O_{0}\right)$ is $\mathfrak{h}$; thus, by the above arguments, the envelope of holomorphy $\hat{\Omega}$ is biholomorphic to $H^{\mathbb{C}} \cong \mathbb{C}^{3}$.

Finally, the maximal convex $\sigma$-invariant subdomain of $\tilde{P}\left(O_{0}\right)$ is $\{(A, B, C) \in$ $\left.\mathfrak{h}: A^{2}+B^{2}<3\right\}=\tilde{P}\left(O_{2}\right)$, where $O_{2}:=\left\{(a, b, c) \in \mathfrak{h}: a^{2}+b^{2}<3 \frac{c^{2}}{\sinh ^{2}(c)}\right\}$. One checks that $O_{2} \subset O_{1}$; thus $\left.\tilde{P}\right|_{O_{2}}$ is injective and $H \cdot O_{2}$ is biholomorphic to $H \cdot \exp _{H^{\mathbb{C}}}\left(i \tilde{P}\left(O_{2}\right)\right)$. Moreover, one has

$$
\begin{aligned}
\exp _{H^{\mathbb{C}}}\left(\left(A^{\prime}, B^{\prime}, C^{\prime}\right)+i(A, B, C)\right) & \\
& =\exp _{H}\left(A^{\prime}, B^{\prime}, C^{\prime}\right) \exp _{H^{\mathrm{C}}}\left(i\left(A, B, C-\frac{1}{2}\left(A^{\prime} B-A B^{\prime}\right)\right)\right) .
\end{aligned}
$$

It follows that

$$
\exp _{H^{\mathbb{C}}}^{-1}\left(H \cdot \exp _{H^{\mathbb{C}}}\left(i \tilde{P}\left(O_{2}\right)\right)=\left\{\left(\operatorname{Im} z_{1}\right)^{2}+\left(\operatorname{Im} z_{2}\right)^{2}<3\right\},\right.
$$

where $\left(z_{1}, z_{2}, z_{3}\right)=\left(A^{\prime}+i A, B^{\prime}+i B, C^{\prime}+i C\right)$ are natural complex coordinates of $h^{\mathbb{C}} \cong \mathbb{C}^{3}$, and this yields the statement.

Remark. Since $\tilde{P}$ is injective on $O_{1}$, the $H$-invariant domain defined by $O_{1}$ is holomorphically separable. As a matter of fact, one may show that $\tilde{P}\left(O_{1}\right)=$ $\mathfrak{h} \backslash\left\{(A, B, C) \in \mathfrak{h}: A^{2}+B^{2} \geq 3, C=0\right\}$, and analogous arguments as above show that such an $H$-invariant domain is not holomorphically convex.

\section{Generalized Heisenberg groups}

Here we apply results of the previous section to generalized Heisenberg groups, exhibiting additional examples of non-Stein maximal domains of existence for the adapted complex structure. We refer to $[\mathrm{BTV}]$ for the basic properties of generalized Heisenberg groups. 
Let $G$ be a generalized Heisenberg group with Lie algebra $\mathfrak{g}$. Consider its abelian subalgebra $\mathfrak{z}:=[\mathfrak{g}, \mathfrak{g}]$ and the subspace $\mathfrak{v}$ orthogonal to $\mathfrak{z}$ with respect to the $G$-invariant metric $(\cdot, \cdot)$ of $G$. Then for all $V+Y \in \mathfrak{v} \oplus \mathfrak{z}=\mathfrak{g} \cong$ $T_{e} G$, the unique geodesic tangent to $V+Y$ at 0 can be explicitly given by $t \mapsto$ $\exp _{G} \circ \varphi_{G}(t, V+Y)$ for a certain real-analytic map $\varphi_{G}: \mathbb{R} \times T_{e} G \rightarrow \mathfrak{g}$ (see [BTV] the theorem on p. 31]).

A straightforward computation shows that $\varphi_{G}$ extends holomorphically on $\mathbb{C} \times$ $T_{e} G$ to $\mathfrak{g}^{\mathbb{C}}$, and arguments analogous to the previous section imply that $\Omega_{G}:=$ $G \cdot O_{G} \subset T G$ is the maximal adapted complexification, where $O_{G}$ is the connected component of

$$
\left\{V+Y \in T_{e} G: \operatorname{det}\left(D \tilde{P}_{G}\right)_{V+Y} \neq 0\right\}
$$

containing 0 , and $\tilde{P}_{G}: T_{e} G \rightarrow \mathfrak{g}$ is given by

$$
V+Y \mapsto l(|Y|) V+\left(1+|V|^{2} m(|Y|)\right) Y .
$$

Here $|\cdot|$ denotes the norm induced by $(\cdot, \cdot)$, and the real-analytic functions $l, m: \mathbb{R} \rightarrow \mathbb{R}$ are defined by

$$
l(t):=\frac{\sinh (t)}{t}, \quad m(t):=\frac{t-\sinh (t) \cosh (t)}{2 t^{3}} .
$$

Now for $V+Y \in \mathfrak{v} \oplus \mathfrak{z}=T_{e} G$ with $Y \neq 0$ and $U+X \in T_{V+Y} T_{e} G \cong T_{e} G$, one has

$$
\begin{aligned}
&\left(D \tilde{P}_{G}\right)_{V+Y}(U+X)=\left.\frac{\partial}{\partial t} \tilde{P}_{G}((V+t U)+(Y+t X))\right|_{t=0} \\
&=l^{\prime}(|Y|) \frac{(Y, X)}{|Y|} V+l(|Y|) U \\
& \\
& \quad+\left(2(V, U) m(|Y|)+|V|^{2} m^{\prime}(|Y|) \frac{(Y, X)}{|Y|}\right) Y \\
&+\left(1+|V|^{2} m(|Y|)\right) X .
\end{aligned}
$$

Note that the equation is written according to the splitting $\mathfrak{v} \oplus \mathfrak{z}$, and since $l(|Y|)$ never vanishes, the $\mathfrak{v}$-part vanishes if and only if

$$
U=-\frac{(Y, X) l^{\prime}(|Y|)}{|Y| l(|Y|)} V
$$

It follows that the central $\mathfrak{z}$-part also vanishes if and only if

$$
\left(1+|V|^{2} m(|Y|)\right) X=|V|^{2} \frac{(X, Y)}{|Y|}\left(2 \frac{l^{\prime}(|Y|)}{l(|Y|)} m(|Y|)-m^{\prime}(|Y|)\right) Y .
$$

In particular, $Y$ and $X$ have to be proportional. Since both sides are homogeneous of degree 1 in $X$, then $\left.(D \varphi)\right|_{(V+Y)}$ is singular if and only if

$$
1+|V|^{2} m(|Y|)=|V|^{2}|Y|\left(2 \frac{l^{\prime}(|Y|)}{l(|Y|)} m(|Y|)-m^{\prime}(|Y|)\right) .
$$

An analogous computation shows that $\left(D \tilde{P}_{G}\right)_{V+Y}$ has maximal rank if $Y=0$; thus equation (5) describes the singular locus of $D \tilde{P}_{G}$. It is remarkable that this identity is independent of the fine structure of the generalized Heisenberg group, e.g., of its dimension or the dimension of its center. In particular, if $H$ is the 3-dimensional Heisenberg group considered in the previous section, equation (5) determines the boundary of $O_{H}=\Omega_{H} \cap T_{e} H$. Using this fact, we are now going 
to show that for a generalized Heisenberg group $G$ there exist many copies of $\Omega_{H}$ embedded as closed submanifolds in $\Omega_{G}$.

Let $G$ be a generalized Heisenberg group and choose non-zero elements $\bar{V}_{1} \in \mathfrak{v}$ and $\bar{Y} \in \mathfrak{z}$. Then there exists an element $\bar{V}_{2} \in \mathfrak{v}$ such that the closed subgroup $\exp _{G}\left(\operatorname{span}\left\{\bar{V}_{1}, \bar{V}_{2}, \bar{Y}\right\}\right)$ is a totally geodesically embedded 3-dimensional Heisenberg group (see [BTV, p. 30]). Denote by $I: H \rightarrow G$ such an embedding, and note that since $\exp _{G^{\mathbb{C}}}: \mathfrak{g}^{\mathbb{C}} \rightarrow G^{\mathbb{C}}$ is a biholomorphism, $I$ extends to a holomorphic embedding $I^{\mathbb{C}}: H^{\mathbb{C}} \rightarrow G^{\mathbb{C}}$ of the universal complexification of $H$ into the universal complexification of $G$ such that the diagram

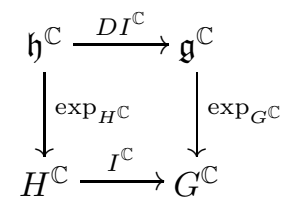

commutes. Now $I: H \rightarrow G$ is totally geodesic; thus, $t \mapsto I \circ \exp _{H} \circ \varphi_{H}(t, v)$ is the unique geodesic of $G$ tangent to $D I(v)$ at 0 for all $v \in \mathfrak{h}$. Then

$$
I \circ \exp _{H} \circ \varphi_{H}(t, v)=\exp _{G} \circ \varphi_{G}(t, D I(v)),
$$

and by the identity principle,

$$
I^{\mathbb{C}} \circ \exp _{H^{\mathbb{C}}} \circ \varphi_{H}(z, v)=\exp _{G^{\mathbb{C}}} \circ \varphi_{G}(z, D I(v))
$$

for all $z \in \mathbb{C}$, since this holds for all $z \in \mathbb{R}$. The commutativity of the diagram

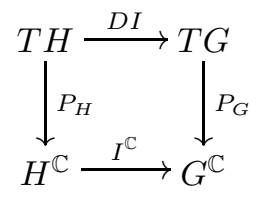

follows. For this, note that, since $I$ is a group homomorphism, then $D I: T H \rightarrow$ $T G$ is $H$-equivariant, i.e., $D I\left(g_{*} w\right)=I(g)_{*} D I(w)$ for all $g \in H$ and $w \in T H$. In particular,

$$
P_{G} \circ D I\left(g_{*} v\right)=P_{G}\left(I(g)_{*} D I(v)\right)=I(g) \exp _{G^{\mathrm{C}}} \circ \varphi_{G}(i, D I(v))
$$

for all $g \in H$ and $v \in T_{e} H$. On the other hand, using equation (6), one obtains

$$
\begin{aligned}
I^{\mathbb{C}} \circ P_{H}\left(g_{*} v\right) & =I^{\mathbb{C}}\left(g \exp _{H^{\mathbb{C}}} \circ \varphi_{H}(i, v)\right)=I^{\mathbb{C}}(g) I^{\mathbb{C}}\left(\exp _{H^{\mathbb{C}}} \circ \varphi_{H}(i, v)\right) \\
& =I(g) \exp _{G^{\mathbb{C}}} \circ \varphi_{G}(i, D I(v)),
\end{aligned}
$$

showing that the above diagram is commutative.

From the equivariance of $D I$ it follows that $D I\left(\Omega_{H}\right)=D I\left(H \cdot O_{H}\right)=I(H)$. $D I\left(O_{H}\right)$. Since $D I$ is isometric and the boundary of $O_{H}$ is defined by equation (5)), which also describes the singular locus of $\tilde{P}_{G}$, one has

$$
D I\left(O_{H}\right) \subset O_{G} \cap \operatorname{span}\left\{\bar{V}_{1}, \bar{V}_{2}, \bar{Y}\right\}, \quad D I\left(\partial O_{H}\right) \subset \partial O_{G} .
$$

Thus $D I\left(\Omega_{H}\right) \cong I(H) \times D I\left(O_{H}\right)$ is closed in $\Omega_{G} \cong G \times O_{G}$.

Furthermore, $D I$ is injective and $P_{H}, P_{G}$ are locally biholomorphic where the adapted complex structure is defined (cf. Theorem[3.2). Thus diagram (7) shows that $D I\left(\Omega_{H}\right) \cong \Omega_{H}$ is a closed complex submanifold of $\Omega_{G}$. Finally, by Proposition 4.3, the domain $\Omega_{H}$ is neither holomorphically separable, nor holomorphically convex. Thus one has 
Proposition 5.1. Let $G$ be a generalized Heisenberg group. Then the maximal adapted complexification $\Omega_{G}$ is neither holomorphically separable, nor holomorphically convex.

\section{REFERENCES}

[A] Agullar, R. Symplectic reduction and the homogeneous complex Monge-Ampère equation, Ann. Global Anal. Geom. 19 (2001), no. 4, 327-353. MR 2002g:53144

[AG] Akniezer, D. N.; Gindikin, S. G. On Stein extensions of real symmetric spaces, Math. Ann. 286 (1990), 1-12. MR 91a:32047

[B] Burns, D. On the uniqueness and characterization of Grauert tubes, Complex analysis and geometry (Trento, 1993), 119-133, Lecture Notes in Pure and Applied Math. 173. MR 97e:32016

[BHH] Burns, D.; Halverscheid, S.; Hind, R. The Geometry of Grauert Tubes and Complexification of Symmetric Spaces, to appear in Duke Math. Journal, math.CV/0109186.

[BTV] Berndt, J.; Tricerri, F.; Vanhecke, L. Generalized Heisenberg Groups and DamekRicci Harmonic Spaces, Lecture Notes in Math. 1598, Springer-Verlag, New York, 1995. MR 97a:53068

[CIT] Casadio-Tarabusi, E.; Iannuzzi, A.; Trapani, S. Globalizations, fiber bundles and envelopes of holomorphy, Math. Z. 233 (2000), 535-551. MR 2001f:32035

[GH] Gilligan, B.; Huckleberry, A. T. On non-compact complex nil-manifolds, Math. Ann. 238 (1978), 39-49. MR 80a:32021

[GS] Guillemin, V.; Stenzel, M. Grauert tubes and the homogeneous Monge-Ampère equation, J. Differential Geom. 34 (1991), no. 2, 561-570 (first part); J. Differential Geom. 35 (1992), no. 3, 627-641 (second part). MR 93e:32018 MR 94e:32032

[Ha] Halverscheid, S. On Maximal Domains of Definition of Adapted Complex Structures for Symmetric Spaces of Non-compact Type, Thesis, Ruhr-Universität Bochum, 2001.

[He] Heinzner, P. Equivariant holomorphic extensions of real analytic manifolds, Bull. Soc. Math. France 121 (1993), 445-463. MR 94i:32050

[Ho] Hochschild, G. The structure of Lie groups, Holden-Day, San Francisco, 1965. MR 34:7696

[LS] Lempert, L.; SzöKe, R. Global solutions of the homogeneous complex Monge-Ampère equation and complex structures on the tangent bundles of Riemannian manifolds, Math. Ann. 290 (1991), 689-712. MR 92m:32022

[L] Loeb, J. J. Pseudo-convexité des ouverts invariants et convexité géodésique de certains espaces symétriques, Séminaire d'Analyse (P. Lelong, P. Dolbeault, H. Skoda), Lecture Notes in Mathematics 1198, Springer-Verlag (1983-84), 172-190. MR 88b:32070

[PW] Patrizio, G.; Wong, P.-M. Stein manifolds with compact symmetric center, Math. Ann. 289 (1991), no. 4, 355-382. MR 92e:32009

[R] Rossi, H. On envelopes of holomorphy, Comm. Pure Appl. Math. 16 (1963), 9-17. MR 26:6436

[Sn] Snow, D-M. Reductive Group Actions on Stein Spaces, Math. Ann. 259 (1982), 79-97. MR 83f:32026

[Sz1] SzöKE, R. Complex structures on tangent bundles of Riemannian manifolds, Math. Ann. 291 (1991), 409-428. MR 93c:53023

[Sz2] SzÖKE, R.Adapted complex structures and Riemannian homogeneous spaces, Complex analysis and applications (Warsaw, 1997) Ann. Polon. Math. 70 (1998), 215-220. MR 99m:53093

Carl von Ossietzky Universität, Fachbereich Mathematik, D-26111 Oldenburg i. O., GERMANY

E-mail address: halverscheid@mathematik.uni-oldenburg.de

Dipartimento di Matematica, Università di Bologna, Piazza di Porta S. Donato 5, I-40126 Bologna, ItALy

E-mail address: iannuzzi@dm.unibo.it 